\title{
AUTOMATIC MONITORING SYSTEM OF ENERGY-SAVING AGRICULTURAL PRODUCTION EQUIPMENT USING WIRELESS SENSOR NETWORK
}

\author{
Hongwei Qi ${ }^{1}$, Haiyan Bai ${ }^{1}$, Haroon Rashid ${ }^{2}$ \\ ${ }^{1}$ School of Computer, Jining Normal University 1, Yili Oriental School, Jining District 2, \\ Ulanqab, Inner Mongolia 012000, China \\ ${ }^{2}$ Department of Structures \& Environmental Engineering, University of Agriculture, Faisalabad, Pakistan \\ Email: qihongw012@126.com
}

\begin{abstract}
In order to resolve the problem of traditional wireless sensor and wired sensor in an operation and to improve the reliability and practicability of the automatic monitoring system of agricultural production equipment; an energy-saving automatic monitoring system of agricultural production equipment (WSN-APEAM) based on wireless sensor network (WSN) is proposed in this study. In this research, sink of WSN-APEAM system is studied. Sensor nodes and actuators of WSNFAEAM system are designed. The MAC layer protocol and routing protocol of WSN-APEAM system and also the calibration engine of WSN-APEAM system are designed and implemented. The upper computer management software of WSN-APEAM system is developed. The security framework of WSN-APEAM system is nominated. In the laboratory of Greenhouse, the system is carried out for test. Experiments show that the service cycle of the sensor networks can take more than three months. Sensor nodes can build multi hop-sensor networks in two minutes by wireless Ad Hoc. The response time of the actuator to various control instructions is less than 15 seconds. All kinds of sensing data can be transmitted to the remote upper computer in real time. To access all kinds of sensing data through the internet/intranet on the upper computer, It can be operated. In this regard, the WSN-APEAM system has the advantages of high stability, low energy consumption, low cost, high accuracy, good robustness, convenient installation, and flexible expansion, which analyze the full coverage monitoring of the monitoring area.
\end{abstract}

Keywords: Wireless Sensor, Automatic Monitoring System For Agricultural Production Equipment, Sink, Calibration Engine, Safety Framework.

\section{Introduction}

The research on agricultural facilities in China started after 1980. Through the continuous efforts of the state, China has made remarkable achievements in the field of agricultural production equipment. However, compared with the developed countries' agricultural production equipment, there are still inadequacies, such as the low level agricultural equipment and facilities, the poor ability to resist natural disasters, the low degree of mechanization, low labor productivity, low scientific and technological content of agricultural production environment monitoring system. These deficiencies are far from meeting the current demand for agricultural automation production. The technology of agricultural facilities and equipment is not standardized and matched, so there are still many shortfalls in the actual use of the system, which cannot achieve the production of agricultural production equipment with a large number of crops. The equipment cultivation technology is not advanced enough, and the equipment management is not refined enough, which leads to the output and quality of crops under this condition of agricultural equipment that cannot be improved further [1].

Wireless sensor networks (WSN) as a distributed sensor network, it's principle is to distribute a large number of sensor nodes in an automatic monitoring range of the system through random delivery. Sensor node consists of communication module, power module, sensor module and controller module. Through wireless communication, layered network communication protocol and distributed algorithm, the wireless network system can be established by efficient self-combination. Sensor nodes can cooperate with each other to make the whole system better. By using sensors of different purposes, the system can detect the environment pressure, temperature, light intensity, humidity, soil composition, noise, moving objects, water quality and other conditions related to crop growth in the monitored area. With the help of gateway, the wireless sensor network can be connected to the 
Internet, and the monitored environment data can be transmitted to the user's client [2]. In terms of agricultural production equipment, wireless sensor network has highly integrated, low-power, low-cost and miniaturized sensor nodes. All sensor nodes are centralized and distributed in the scope of production equipment, to achieve the purpose of monitoring the real-time crop growth environment.

Comparing with the traditional sensor, the wireless sensor network has the characteristics of strong flexibility, high accuracy, strong reliability, self-organization ability and high efficiency. Consequently, it can build effectively for the shortcomings of self-organized multi hop wireless transmission and the collection of environmental monitoring data of agricultural production equipment [3].

Therefore, in this study, WSN based an automatic monitoring system for energy-saving agricultural production equipment (WSN-APEAM) is developed. The system has the advantages of convenient installation, strong stability, low cost, good robustness, small energy consumption, flexible expansion and high accuracy. It can be applied in nursery, greenhouse and other areas to analyze the remote real-time automatic monitoring of the internal environmental conditions of these areas and to resolve the problems of traditional wireless sensors and wired sensors.

\section{Method}

\subsection{Sink design and implementation of WSN- APEAM system}

At present, many researchers have studied the interconnection technology between WSN and backbone network and studied various Interconnection Technologies between WSN and Internet. All interconnection technologies can be divided into three categories: all IP mode, protocol bearing mode and protocol conversion mode [4]. Each of Interconnection Technologies has its advantages and disadvantages, but it is challenging to meet all the demands of WSN-APEAM system. Therefore, in this research, a new interconnection technology, VIP bridge, is proposed.

As shown in Figure 1, VIP bridge is composed on knowledge base, query engine, $\mathrm{T} \rightarrow \mathrm{S}$ translator, packet analyzer, mapping table exchanger, $\mathrm{S} \rightarrow \mathrm{T}$ translator. Packet analyser and packet translator analyze and translate the data from IPv4 network and WSN respectively, therefore, two packet formats can transform each other. After analyzing the data package, the valid data package is sent to the query engine, which extracts the useful information from the knowledge base on form the required data package format. The function of the mapping table exchanger is to integrate the mapping tables of VIP bridges by exchanging the mapping table information among them.

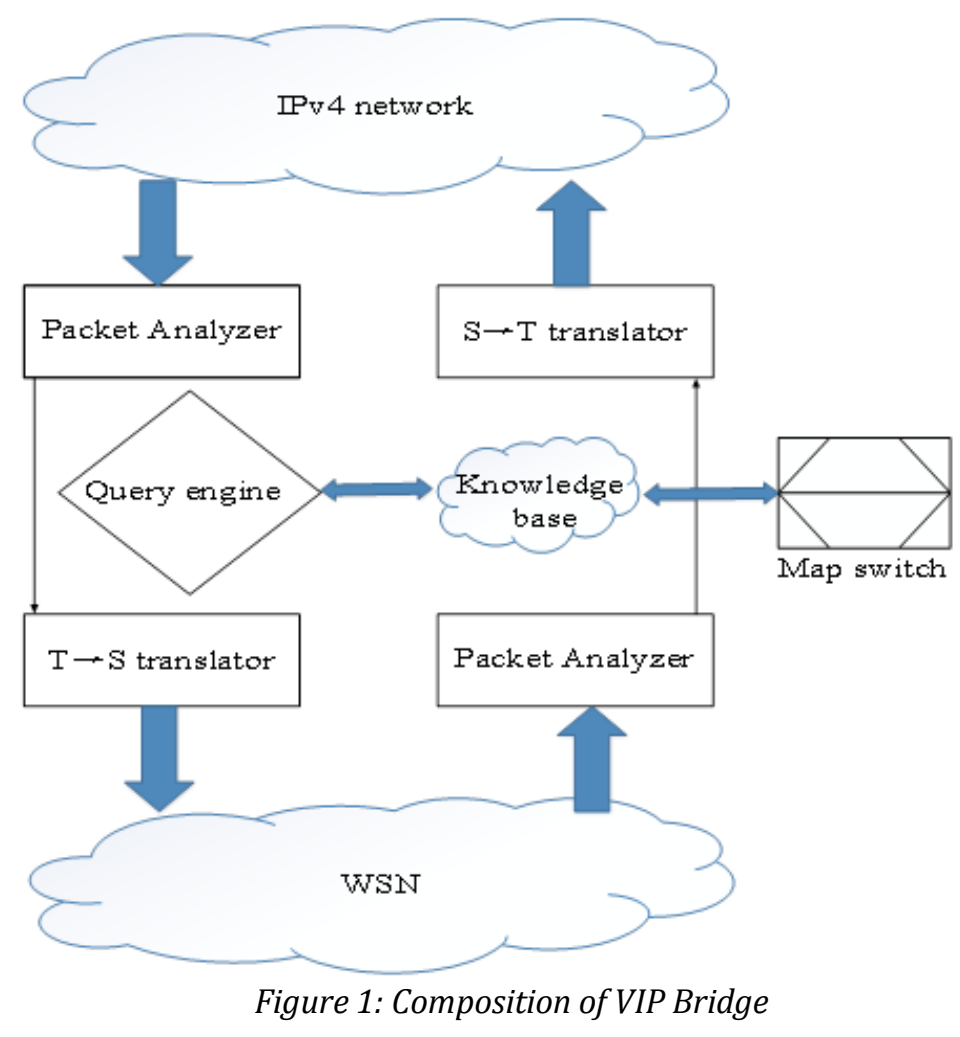

According to the application characteristics of WSN-APEAM system, when building Sink, MCU + CC2420 is taken on the basis [5] of this report, and the realization idea of sink in WSN-APEAM system is proposed. As shown in Figure 2, the design idea is shown. 


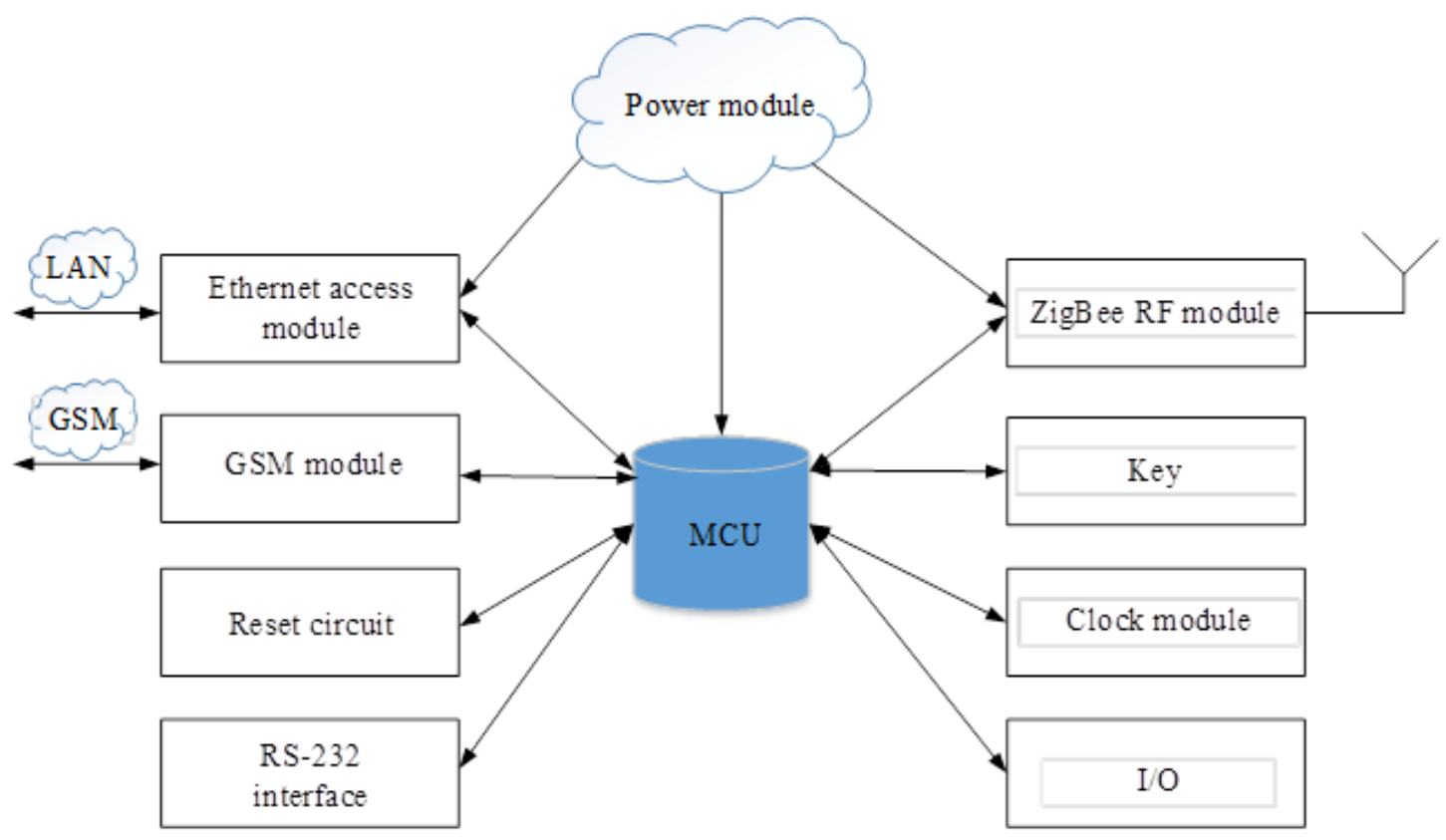

Figure 2: Sink structure of WSN-APEAM system

The price, start-up time, receiving and transmitting power, energy consumption, data transmission rate and other aspects are fully balanced. At the same time, considering that TinyOS has included CC2500 driver support, then when the system is used, Ethernet controller CS8900A with low price and energy consumption is selected for Ethernet interface, 2.4GHz RF chip CC2500 with low price and energy consumption are used for ZigBee RF module, and TIMSP430F5418 microcontroller are used for MCU (Single Chip Microcomputer).

\subsection{Node design and implementation of WSN-APEAM system}

(1) Sensor node design of WSN-APEAM system

With the increasing level of artificial intelligence in the automatic monitoring system of agricultural production equipment environment, types and quantity of sensors are used in the system will be strengthened it continuously, which cause severe problems such as compatibility and interoperability between sensors and communication networks. In order to effectively avoid the above problems, in the design of WSN-APEAM system, sensor nodes follow IEEE Std 1451.0TM-2007 and IEEE Std 1451.5TM2007. The types of sensors that are used: 5TE soil conductivity sensor, SHT75 temperature sensor, 5TE soil temperature sensor, SHT75 humidity sensor, 5TE soil humidity sensor. CGs-3100 carbon dioxide sensor and TBQ-6 illuminance sensor are used to measure the stem diameter change of Ecomatikdd.

(2) Sensor node structure of WSN-APEAM system

In this study, during the development of WSNAPEAM system, sensor nodes are designed by wired sensor board.
USART (Universal Synchronous Asynchronous Receiver Transmitter) is used to realize communication between the sensor board (TIM) and the processor board (NCAP).

In the sensor board, it's main components are signal regulator, ADC / DAC and related auxiliary circuits. The sensor board and the processor board share the same microprocessor. Some sensors, such as humidity sensor, illuminance sensor and temperature sensor, are directly integrated on the sensor board. Other sensors shall be independent of the outside of the sensor board, and connected to the corresponding interface of the sensor board through their respective cables.

In the processor board, the following modules are mainly integrated: TIMSP430F5418 microcontroller processor module, $2.4 \mathrm{GHz}$ RF chip CC2500 wireless communication module, energy supply module and some are related to auxiliary circuits.

(3) TEDS design of sensor nodes

TEDS, as the core component of IEEE1451 intelligent sensor, is also one of the essential components to analyze the plug and play operation between processor board and sensor board [6]. Therefore, it is necessary to define required TEDS for various sensor nodes in WSN-APEAM system.

(4) Design and implementation of executive mechanism of WSN-APEAM system

In WSN-APEAM system, the main function of the actuator is to control the DC solenoid valve of each physical device in the system, so that the system can automatically control each physical device. Compared with the sensor node, the actuator has basically the same structure except that there is no sensor board (as shown in Figure 3). 


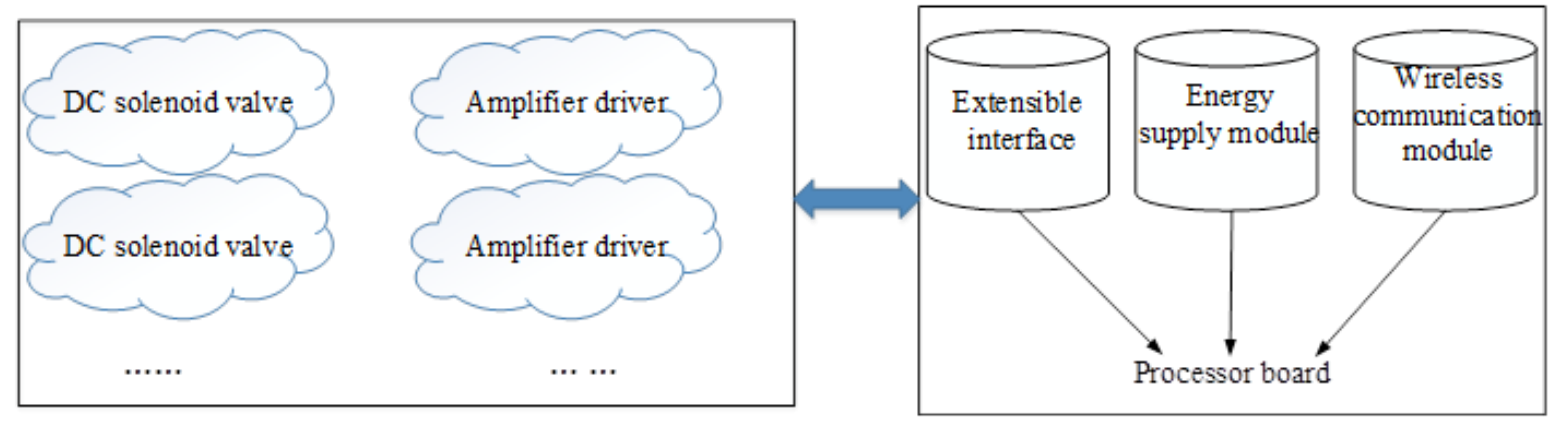

Figure 3: Hardware framework of actuator

\subsection{MAC layer protocol and routing protocol of WSN-APEAM system}

(1) Design and implementation of MAC protocol in WSN-APEAM system

Sensor-MAC (S-MAC) is a MAC layer protocol based on CSMA (Carrier Sensor Multiple Access with Collision Avoidance) random competition. It's function is to reduce the energy consumption of sensor nodes, so that it is used to achieve the purpose of energy saving. In order to achieve this goal, S-MAC protocol uses the following methods. Periodic listening/sleeping mechanism is used to eliminate idle listening; virtual carrier monitoring, physical carrier listening and instant communication/communication switch system are used to reduce conflicts in mobile communication access network. Nodes that may cause blocking to enter sleep state after listening to the "request sending" or "clear sending packets" to avoid crosstalk. Message transmission mechanism can effectively support prolonged message sending [7].

According to the use characteristics of agricultural production equipment, based on S-MAC protocol, a dynamic scheduling method suitable for network load is adopted [8]. In this study, a more advanced MAC layer protocol, S-MAC + protocol, is constructed. Compared with the traditional S-MAC protocol, this protocol is improved into two aspects.

The periodic transmission of synchronous frames is integrated into the CTS transmission cycle is shorten the monitoring cycle. The dynamic duty cycle mechanism is used to enhance the energy efficiency of nodes and systems.

(2) Design and implementation of routing protocol in WSN-APEAM system

The routing protocol of WSN-APEAM system is based on MCFA (minimum cost forwarding algorithm for large sensor networks). According to the use characteristics of agricultural production equipment, two aspects of MCFA algorithm have been modified and improved.
Whenever, a node finds a better forwarding node to the root node, it updates the parent node. Dynamic routing information can be obtained by this method.

The data of MCFA algorithm is transmitted through broadcast. The intermediate node determines whether discard data or receive \& forward data. According to whether the difference between the data cost and the cost has already spent matches the value in its own cost field. However, this method increases the amount of data and computation in network transmission. In order to resolve this problem in WSN-APEAM system, unicast is used to send all kinds of sensor data, this is used when nodes need to send data, it directly sends the data to it's current parent node.

\subsection{Calibration engine of WSN-FAEAM system}

(1) Sensor node correction and compensation technology

Due to the interference of the following aspects, the sensor nodes of WSN-APAM system may produce detection errors. Sensor nodes have non-linear characteristics. The response speed and other dynamic characteristics of sensor nodes are not ideal. In the process of measurement and control, sensor nodes will be interfered by external environmental factors such as temperature [9]. Therefore, the WSN-APEAM system in this study needs compensation and correction before it is applied to the actual agricultural production. The calibration and compensation technology of sensor nodes can be divided into two categories: static calibration and compensation technology and dynamic calibration and compensation technology.

(2) The mathematical model of IEEE1451 calibration engine

IEEE1451 uses the static correction compensation technology, and takes the polynomial model as the mathematical model of the correction engine:

$$
f\left(x_{1}, x_{2}, \mathrm{~L} x_{n}\right)=\sum_{i=0}^{D_{1}} \sum_{j=0}^{D_{2}} \mathrm{~L} \sum_{p=0}^{D_{n}} C_{i, j, \mathrm{~L}, p}\left[x_{1}-h_{1}\right]^{i}\left[x_{2}-h_{2}\right]^{j} \mathrm{~L}\left[x_{n}-h_{n}\right]^{p}
$$


On the right side of the equation are polynomials of many variables. In this equation, $\mathrm{n}$ represents the number of channels of the calibration engine model;

$\mathrm{x}_{\mathrm{n}}$ represents the output of the sensor or the input of the actuator in channel $\mathrm{n} ; C_{i, j, \mathrm{~L}, p}$ represents the coefficient of the polynomial; $D_{n}$ represents the number of times of the input/output $\mathrm{x}_{\mathrm{n}} ; \mathrm{h}_{\mathrm{n}}$ represents the segmented offset of the input/output $\mathrm{x}_{\mathrm{n}}$.

If $z_{n}=x_{n}-h_{n}$, the equation (1) can be expressed as:

$$
y=\left(A_{0}+A_{1} z_{1}+A_{2} z_{1}^{2}+\mathrm{L}+A_{i} z_{1}^{i}\right)\left(B_{0}+B_{1} z_{1}+B_{2} z_{2}{ }^{2}+\mathrm{L}+B_{j} z_{2}{ }^{j}\right) \mathrm{L}\left(N_{0}+N_{1} z_{n}+N_{2} z_{n}{ }^{2}+\mathrm{L}+N_{p} z_{n}{ }^{p}\right)
$$

For the 2 input/output cases, the correction function can be simplified as:

$$
y=A_{0} B_{0}+A_{1} B_{0} z_{1}+A_{2} B_{0} z_{1}^{2}+A_{3} B_{0} z_{1}^{3}+\mathrm{L}+A_{i} B_{0} z_{1}^{i}+A_{0} B_{1} z_{2}+A_{1} B_{1} z_{1} z_{2}+A_{2} B_{1} z_{1}^{2} z_{2}+\mathrm{L} A_{i} B_{j} z_{1}^{i} z_{2}{ }^{j}
$$

If the number of times of each input is 1 , the correction function can be expressed as:

$$
y=C_{0,0}+C_{0,1}\left(x_{2}-h_{2}\right)+C_{1,0}\left(x_{1}-h_{1}\right)+C_{1,1}\left(x_{1}-h_{1}\right)\left(x_{2}-h_{2}\right)
$$

(3) Modeling sensor nodes by interpolation

Using the interpolation method to model the sensor, the process is as follows. The sensor nodes are calibrated to obtain the calibration values. Appropriate interpolation methods are used to construct and calculate interpolation polynomials. The interpolation polynomial is obtained from the mathematical model of sensor node, which is also known as the mathematical model of sensor node correction engine.

At present, the widely used polynomial interpolation methods include spline interpolation equation, Newton interpolation equation, Hermit interpolation equation and Lagrange interpolation equation. Because the piecewise cubic spline can make up for the short-comings of other interpolation methods [9], in the process of sensor node correction and compensation, the piecewise cubic spline is used as the mathematical model of sensor node correction engine is studied in it.

(4) Calibration TEDS design of sensor nodes in WSN-APEAM system

In order to improve the accuracy of SHT75 humidity sensor with the development of WSNAPEAM system, the nodes of humidity sensor are calibrated.
Therefore, in this study, the SHT75 humidity sensor is calibrated to get the input-output characteristics of different ambient temperatures, that is, calibration test data. On this basis, the calibration engine model of the humidity sensor node is constructed.

After building the calibration engine model of the humidity sensor node, it's calibration TEDS is defined [10-14].

\subsection{The upper computer management software of WSN-APEAM system}

The upper computer management software of WSN-APEAM system consists of two modules: background management software and data management software.

The background management software of WSNAPEAM system consists of three parts: transmission network, background management software and WSN, as shown in Figure 4. There are four sub modules: background component, database, user graphic interface and data processing engine, as shown in Figure 5.

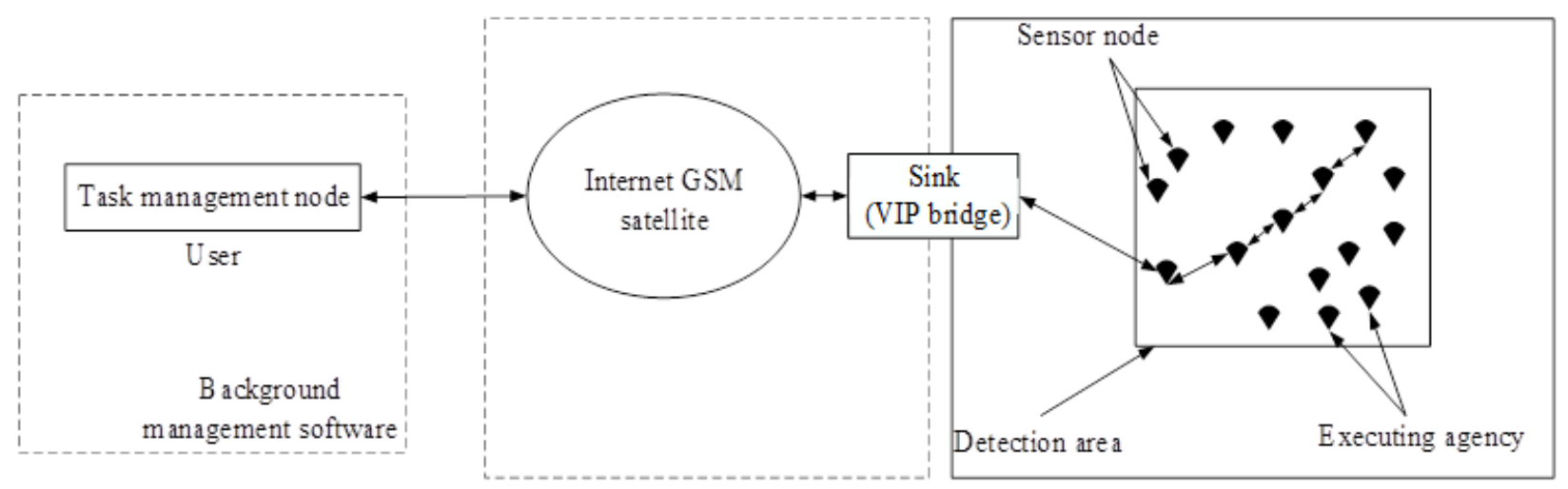

Figure 4: Background management software structure of WSN-APEAM system 


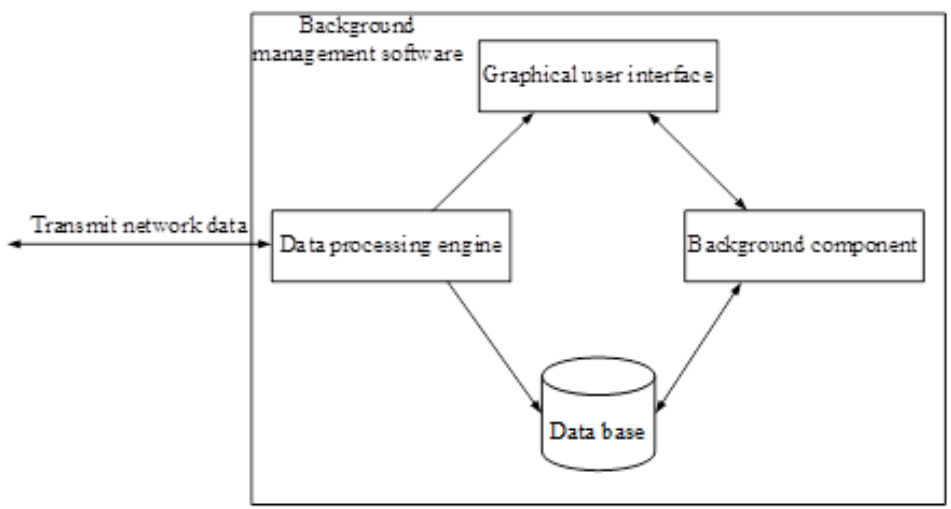

Figure 5: Background management software composition of WSN-APEAM system

Data management software is composed of data acquisition module, data processing module, parameter setting module, data management module, and control output module. This software is used to realize data correction, data fusion, data management, data display and data query, remote query, and interactive operation functions.

\subsection{Security framework of WSN-APEAM system}

As a network system, WSN-APEAM has security problems, so that the security of the system must be improved before the actual operation. WSN-APEAM system consists of Sink, user network, backbone network, task management network or sensor network. As shown in Figure 6, the logical relationship between components is shown.

Based on fully absorbing existing research results, in this study, the following WSN network security dimensions are proposed: data integrity, data confidentiality, effectiveness, access control, authentication, data confidentiality, non-repudiation and communication security. In wireless sensor networks, there are a variety of security threats, which are divided into routing and node related threats.

In this study, the network security deployment and security mechanism of WSN are proposed by absorbing the relevant literature.

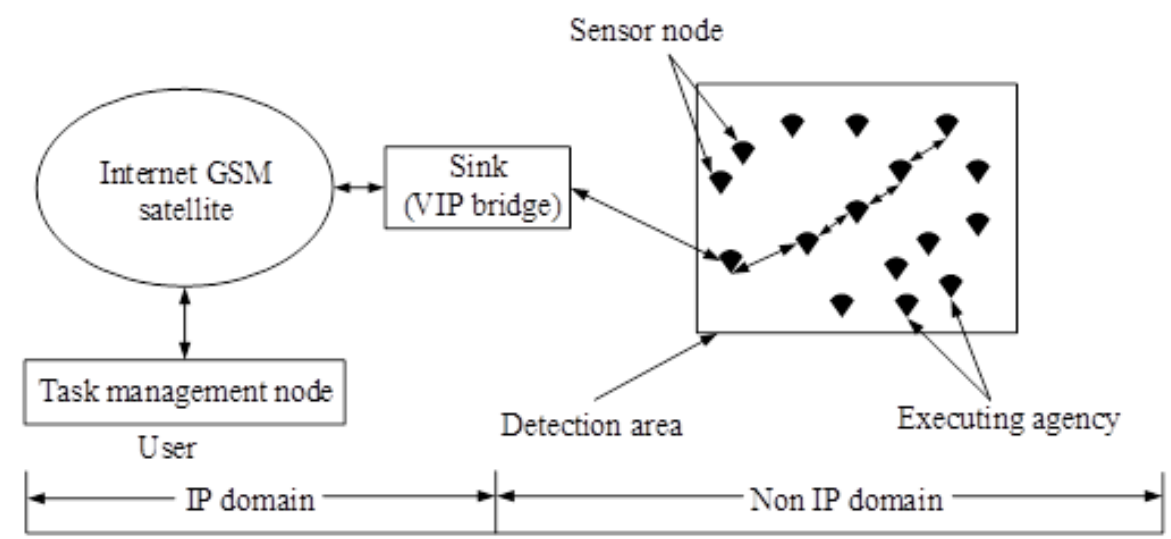

Figure 6: Relationship between WSN-FAEAM system components

\subsection{Test WSN-APEAM system}

In order to test the feasibility and excellence of the research and development system in this study, the experiment is carried out in the intelligent greenhouse of the laboratory. The test scheme is as follows:

In the laboratory of the greenhouse, 3 actuators, 2 sink nodes and 25 sensor nodes are placed.

The actuator is used to control the DC solenoid valve connected with itself, so that the physical equipment such as irrigation equipment can realize automatic control. The sensor calibration engine runs on the same platform as the upper computer management software, and the computer is located in a remote laboratory. Sink is used for the conversion between sensor network protocol and GSM protocol or Ethernet protocol, that is used to transmit sensor data to remote host through GSM or local area network.

The computer running the correction engine and the upper computer management software also plays the role of a network server. Users can access and control the server through the Internet. During the experiment, the data of sensor nodes are collected in every 30 minutes. 
The execution mechanism only works when receiving relevant instructions from the upper computer and remains dormant for a rest of time.

\section{Results and Discussion}

Through the experiment of WSN-APEAM system, WSN-APEAM system can not only meet the basic needs of an automatic monitoring system of agricultural production equipment, but also has the following advantages:

(1) Excellent energy efficiency

The energy consumption of the system can be reduced by using the sleep mechanism and controlling the data sampling interval. Two lithium batteries (each with a capacity of $1200 \mathrm{MA}$ ) can be used for more than three months after charging once.

(2) The ability to quickly self-organize networks

The average communication radius between sensor nodes in this study is about 16 meters. After the arrangement, the sensor nodes can establish the multi hop sensor network in wireless Ad Hoc mode within two minutes. It takes less than one minute to delete or add sensor nodes.

(3) High measurement and control accuracy

When the system works stably, the response time of the actuator to various control instructions is less than 15 seconds, and the bit error rate of the data is very low. After data fusion of sensor data, the data error rate of sensor nodes is about $0.1 \%$, as shown in Figure 7 and Figure 8.

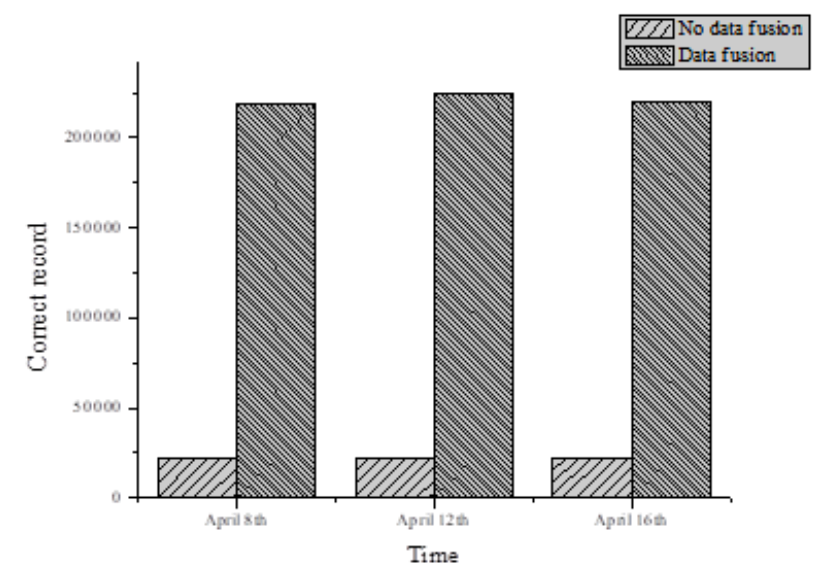

Figure 7: Comparison of correct sensor records before and after data fusion

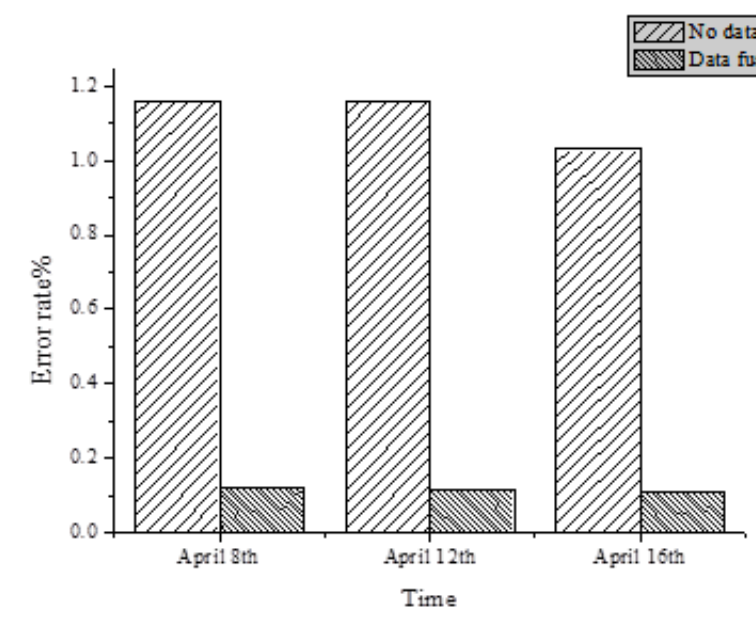

Figure 8: Error rate comparison of WSN-APEAM system before and after data fusion

(4) Good stability

All sensor data is transmitted to sink through multi hop mode, and then through Sink's GSM module or Ethernet access module, the data can be transmitted to the client in a real time. The upper computer management software can accurately analyse and transform all kinds of data and instructions can be calibrated and integrated all kinds of sensor data, and can also store and analyse all kinds of sensor data according to the required format. The actuator can precisely control the DC solenoid valve with the command of the upper computer.

(5) Remote access and remote operation capability

Users can use the Internet to access all the sensor data of the upper computer and can also control the upper computer based on the Internet. Moreover, 
based on the Internet, it can approach the remote operation of the on-site actuator, so it will easy to control the on-site physical equipment.

To sum up, WSN-APEAM system can realize the automatic arrangement and self-organized transmission of data collection nodes, and it can be applied to agricultural production equipment to make up for the shortcomings of the traditional greenhouse monitoring system. The system can enable people to achieve the environmental information of crop growth in the greenhouse at any place and time through Internet/intranet, such as soil temperature, environmental temperature, $\mathrm{CO}_{2}$ concentration, soil humidity, environmental humidity, soil conductivity and other physical environment changes. So, it is used to achieve the purpose of remote, real-time and accurate monitoring of crop growth environment. Compared with the traditional sensor fixed-point layout, the system has the advantages of high stability, low energy consumption, low cost, high accuracy, good robustness, convenient installation and flexible expansion, and can realize the full coverage monitoring of the monitoring area.

\section{Conclusions}

In this study, WSN-APEAM is developed based on wireless sensor network. The system has many functions and can meet the needs of practical applications. Compared with the traditional highquality sensor method with special signal processing, the system has the advantages of high stability, low energy consumption, low cost, high accuracy, good robustness, convenient installation and flexible expansion, and can realize the full coverage monitoring of the monitoring area. After further improvement, WSN-APEAM system can be applied to greenhouse, farmland, nursery and other areas to realize remote, real-time and automatic monitoring of biological growth environment. However, at the same time, the system still has some shortcomings, such as insufficient safety, Sink is difficult to meet the practical needs of future facilitative agricultural production; so it is necessary to continue to create conditions for relevant research to improve the performance of the system.

\section{References}

[1] Suma N, Samson S R, Saranya S, et al. IOT based smart agriculture monitoring system. International Journal on Recent and Innovation Trends in computing and communication, 2017, 5(2), pp. 177-181.

[2] Yeo U, Lee I, Kwon K, et al. Analysis of Research Trend and Core Technologies Based on ICT to Materialize Smart-farm. Protected Horticulture and Plant Factory, 2016, 25(1), pp. 30-41.
[3] Tzounis A, Katsoulas N, Bartzanas T, et al. Internet of Things in agriculture, recent advances and future challenges. Biosystems Engineering, 2017, 164, pp. 31-48.

[4] Ejeh C J, Akhabue G P, Annan B E. Evaluating The Impact of Unsteady Viscous Flow And Presence Of Solid Particles On Pipeline Surfaces During Crude Oil Transport. Acta Mechanica Malaysia, 2019, 2(2): 20-27, DOI: 10.26480/amm.02.2019.20.27

[5] Umamaheswarrao P, Ranga Raju D, Suman KNS and Ravi Sankar B. Topsis Based Optimization Of Process Parameters While Hard Turning Of Aisi 52100 Steel. Acta Mechanica Malaysia, 2019, 2(2), 28-31, DOI: 10.26480/amm.02.2019.28.31

[6] Soh Hui G, Shahreen K, Rohayanti H, Zalmiyah Z, Hairulnizam M, Azizul Azhar R, Mohd Farhan M F, Mohamad Aizi S. Online Activity Duration Management System for Manufacturing Company. Acta Electronica Malaysia, 2019, 3(2), 01-08.

[7] Hesham E, Qasem Abu A H, Hasan A A, Mohamed A N, Sultan Al-Muhaisen. A Schematic Design of HHO Cell as Green Energy Storage. Acta Electronica Malaysia, 2019; 3(2), 09-15.

[8] Hassan K, Muhammad A, Syed Tauseef M. Numerical Solution of Fractional Boundary Value Problems By Using Chebyshev Wavelet Method. Matrix Science Mathematic, 2019, 3(1), 13-16.

[9] Srbinovska M, Gavrovski C, Dimcev V, et al. Environmental parameters monitoring in precision agriculture using wireless sensor networks. Journal of cleaner production, 2015, 88, pp. 297-307.

[10] Wang J, Niu X, Zheng L, et al. Wireless midinfrared spectroscopy sensor network for automatic carbon dioxide fertilization in a greenhouse environment. Sensors, 2016, 16(11), pp. 1941.

[11] Sarkar P J, Chanagala S. A survey on iot based digital agriculture monitoring system and their impact on optimal utilization of resources. Journal of Electronics and Communication Engineering (IOSR-JECE), 2016, 11(1), pp. 1-4.

[12] Huh J H. PLC-based design of monitoring system for ICT-integrated vertical fish farm. Humancentric Computing and Information Sciences, 2017, 7(1), pp. 20.

[13] Nellore K, Hancke G. A survey on urban traffic management system using wireless sensor networks. Sensors, 2016, 16(2), pp. 157.

[14] Modieginyane K M, Letswamotse B B, Malekian $\mathrm{R}$, et al. Software defined wireless sensor networks application opportunities for efficient network management: A survey. Computers \& Electrical Engineering, 2018, 66, pp. 274-287. 